\title{
Feature
}

\section{An exploration of social justice intent in photovoice research studies from 2008 to 2013}

\author{
Marie-Anne Sanon, ${ }^{a}$ Robin A. Evans-Agnewb and Doris M. Boutainc \\ aHealth Promotion Risk Reduction (Division II), School of Nursing, University of Michigan, Ann Arbor, MI, bNursing and \\ Healthcare Leadership, University of Washington, Tacoma, WA, cPsychosocial and Community Health, University of \\ Washington School of Nursing, Seattle, WA, USA \\ Accepted for publication 15 January 2014 \\ DOI: | 0.1 | | | /nin. 12064
}

SANON M-A, EVANS-AGNEW RA and BOUTAIN DM. Nursing Inquiry 2014; 21: 212-226

An exploration of social justice intent in photovoice research studies from 2008 to 2013

In an age where digital images are omnipresent, the use of participant photography in qualitative research has become accessible and commonplace. Yet, scant attention is paid to the social justice impact of photovoice amongst studies that have used this innovative method as a way to promote social justice. There is a need to review this method to understand its contributions and possibilities. This literature review of photovoice research studies (i) explores whether authors implicitly or explicitly related the methodologies to their aims of promoting social justice (methodology-method fit) and (ii) outlines the social justice research impact of photovoice findings using the framework of social justice awareness, amelioration and transformation. PubMed, Scopus, PsycINFO and Web of Science databases were searched from the years 2008-13 using the following keywords: photovoice; photonovella; photovoice and social justice; and photovoice and participatory action research. Of the 30 research studies reviewed, only thirteen identified an underlying methodology guiding the photovoice method. The social justice impacts emphasized were more related to social justice awareness $(n=30)$ than amelioration $(n=11)$ or transformation $(n=3)$. Future researchers using photovoice as a way to promote social justice are encouraged to assess and plan for the social justice impact desired.

Key words: critical methodologies, photography/photovoice, social justice impact.

The last two decades witnessed an explosion in the use of participant - photography or photovoice by qualitative health researchers. Specifically within nursing research, photovoice is increasingly being adopted by nurse researchers as an innovative method to explore individuals' lived experiences specific to diseases and other phenomenological inquiries (Poudrier and Mac-Lean 2009; Plunkett, Leipert and Ray 2013). Nurse researchers have acknowledged the promise of photovoice to facilitate individual empowerment and community involvement alongside the demystification of oppressive patterns and systems (D'Alonzo 2010; Ponic, Reid and Frisby 2010).

Correspondence: Marie-Anne Sanon, Post-Doctoral Fellow, 400 North Ingalls, Room 3356, Ann Arbor, MI, 48 109, USA. E-mail: <sanon@umich.edu>
Developed by Wang and Burris (1994, 1997), photovoice was first used with the theoretical underpinnings of Freire's critical consciousness and feminism (Freire 1973; Weiler 1988). Wang proposed photovoice as a method for those marginalized to document their experiences and comment on the social and political forces that influenced those experiences.

There were three original reasons proposed for the use of photovoice. First, it could record and document the strengths and weaknesses of a community. Second, it could empower individuals by providing a platform for group and community discussions. Third, critical dialogues from photovoice - geared towards community improvement - could be used to influence policy making to promote systemic change (Wang 1999). 
However, since its introduction, several researchers (including nurse researchers) have critiqued the photovoice method. Critiques of photovoice have focused on either ethical dilemmas (Wang and Redwood-Jones 2001; Prins 2010; Allen 2012; Evans-Agnew, Sanon and Boutain in press) or on methodological and technical challenges (Royce, ParraMedina and Messias 2006; Novek and Morris-Oswald 2012; Evans-Agnew et al. in press). Despite these concerns, these critiques reaffirm Wang's (Wang and Pies 2004; Wang et al. 2004) contention that the principal goal of this method is to influence policy change and promote social justice. However, the extent to which these impacts occur as a result of research using photovoice requires further study.

This article aims to advance the use of the photovoice method by conducting a historical review of its use related to social justice. There are two specific purposes for this article. This article explores how the authors implicitly or explicitly related the methodologies to the historical photovoice aims of social justice. In this paper, we define this as methodology-method fit. This article also maps the research impacts using a framework of social justice awareness, amelioration and transformation. Impact in this article refers to how the research findings were used to promote change either at the individual level or in the systems, environment or policy realms.

This article is organized in four major sections. First, we provide a view of prior critiques of photovoice as a method. Second, we describe the framework we use to critique the literature, focused on methodology-method fit and social justice approaches. Third, we outline the literature review method used to retrieve studies reviewed in this article. Fourth, we analyze whether the authors implicitly or explicitly reported how their methodologies were driven by the goal of promoting social justice (methodology-method fit). Fifth, we explore the social justice impact of the research studies relating to awareness, amelioration and transformative action. Last, we summarize the implications for future research.

\section{PRIOR CRITIQUES OF THE PHOTOVOICE METHOD}

Extensive reviews of photovoice were completed in 2009 (Hergenrather et al. 2009) and 2010 (Catalani and Minkler 2010). However, neither review focused on the extent to which the photovoice findings promoted social justice impact. Instead, the use and possibilities of the photovoice method was the primary focus.

Hergenrather et al. (2009) reviewed 31 articles to determine how photovoice promoted individual and community change. Individual and community change referred to increased individual awareness and participation as change agents and physical improvements within the community, respectively. They reported inconsistencies in the photovoice process amongst the studies. They recommended the need for researchers to identify both researchers' and participants' role in all aspects of the project.

Catalani and Minkler (2010) conducted a review of 37 photovoice studies using Wang's photovoice approach to explicate the positive relationship amongst community involvement, individual empowerment, community asset recognition and action towards policy change. Community involvement activities were defined as those including interactive activities such as training, discussion and documentation. Their critique of photovoice studies preceded the year of 2008. The critique focused on how the photovoice process was undertaken (e.g. recruitment, sample size, sample characteristics and training), including a particular focus on the elements of participant engagement (e.g. 'participant involvement' and 'empowerment'). Inconsistencies in rigour and fit between the method and the particular health concern of interest were noted. Although most of the studies (60\%) used the word 'impact,' impact referred to community member involvement and their experiences of individual empowerment and awareness. No observations were made about other aspects of social justice impact (for example, in addition to individual empowerment, including policy change) as a result of using the photovoice method. We extend Catalani and Minkler's (2010) analysis to review and describe the social justice impact of photovoice research and also by extending the discussion on methodology and method fit for articles published since 2008.

In the next section, we will begin with discussions about methodology-method fit and the social justice framework. These two discussions will anchor our analysis of the research studies retrieved. The discussion provides more information about the theories used to analyze the studies.

\section{ANALYZING METHODOLOGY —METHOD FIT}

The identification of methodologies guiding research methods is of upmost importance (Crotty 1998; Chenail 2009; Mack 2010). Methods that are intended for justice promotion must be guided by justice-oriented methodologies and philosophical underpinnings (Evans-Agnew et al. in press). Researchers have been called upon to be transparent about their epistemological underpinnings, research approach (methodology) and their chosen tools to execute the research process (methods; Crotty 1998; Chenail 2009). 
Hansen-Ketchum and Myrick (2008) support this notion with respect to photovoice as they argued that peoples' existence/reality (ontology), belief about how knowledge is acquired (epistemology) and their approach to developing knowledge (methodology) most notably shape their decision about research methods. Thus, when aiming to use a methodology that attends to social justice, it is important to have clarity about the social justice intentions.

\section{Analyzing Social Justice Impact}

Definitions of social justice have focused on the equal distribution of benefits and burdens in society (Redman and Clark 2002; Boutain 2011) and promoting change in society focusing on social relationships and institutions (Drevdahl et al. 2001). Buettner-Schmidts and Lobo synthesized the definition of social justice as the "full participation in society and the balancing of benefits and burdens by all citizens, resulting in equitable living and a just ordering of society' (2012, 954). Researchers who use a social justice framework emphasize moral obligation and citizens' rights (Boutain 2011) and a focused agenda of direct change to minimize subordination and vulnerability to social injustice (Kirkham and Anderson 2002).

A social justice framework could benefit researchers in photovoice design, given the original goals for photovoice use. Despite the extent of photovoice studies, an evaluation of the social justice impact of research using the photovoice method has not been completed. In this review, we employ the social justice insights described by Boutain (2011) that includes three major ways of focusing on social justice impact. Namely, we will focus on social justice awareness impacts, social justice ameliorative impacts and social justice transformative impacts related to photovoice use in research studies. This framework is selected as it describes a variety of ways to delineate social justice impacts. Each framework component is described in more detail below.

\section{Social Justice Awareness}

Social justice awareness focuses on making burdens and benefits more apparent with attention to relationships of power. Awareness impacts inspire cognitive, emotional or intellectual insights on the part of individuals or groups. It can promote constant questioning of the influence of systems of oppression in creating privilege, marginalization and health. It calls for contextual consideration on the part of the researcher to begin to grasp different aspects of a phenomenon. Social justice awareness is ongoing and is never fully met as it is 'temporal and [is] dependent on [one's] frame of reference' (Boutain 2011, 51).

In a photovoice project, findings may be used to help researchers, participants and audience members reach new understanding about systems of oppression. The photovoice findings may show connections between health and society anew. Findings can provide visuals of life realities. Thus, visual senses can be heightened to identify the vulnerable and privileged and the factors leading to the disparities. Although awareness is needed as an initial process of consciousness raising to understand issues of power, privilege and health compromise, this impact is most focused on increasing sensibilities for individuals or groups about issues using the photovoice approach.

\section{Social Justice Amelioration}

Social justice amelioration impact involves actions to mitigate the immediate factors leading to the unjust conditions. Social justice amelioration does not provide long term remediation of the health and social injustices. Instead, the social justice impact is an immediate reaction to address acute and emergent issues that are symptoms of oppression or power imbalances which result in vulnerability and health compromise.

Photovoice findings may inspire remedial reactions by those who are present. Or the photographs may be presented in a way to anchor reactive discussions about actions needed. Reactions may be short-term or long-term but will centre on addressing symptoms of oppression and not address systems of power which lead to oppression or health vulnerabilities. Often, these reactions do not deeply consider how to change one's power position to subvert participation in oppressive relationships. For example, the reactions may centre on how to address the issues presented by those who have taken the photos. The discussion does not focus on the conditions which gave rise to the contexts or issues photographed.

\section{Transformative Action}

Unlike social justice amelioration, which does not prevent the problem from recurring, an impact focused on social justice transformation aims to address the issue at its roots. The goal of transformative impact is to promote change in systems of oppression in ways of being, ways of interacting and ways of governing. These changes can be explicitly shown at the systems, governance or policy levels to eliminate or minimize the underlying factors leading to power imbalances and unjust conditions. Systems-level change would show a 
difference in relationships between institutions or sectors. Policy change, an alteration in the governance of how systems are operated, may occur at the institutional or public regulatory levels.

Transformative impacts can involve new policy development or changes in existing policies. For example, photovoice findings may inspire changes in systems of care to address patterns of poor health outcomes. Additionally, photovoice findings which show unequal, unjust or uneven applications of regulations in addition might prompt a more extensive policy review. Thus, research impacts using photovoice would most likely focus on system, policy or environmental changes.

\section{METHODS}

\section{Sampling}

Two research questions guided our review of the literature. Those questions were as follows: Did study authors who use the photovoice method with the intent to promote social justice describe their methodology with a justice-based orientation (methodology-method fit)? How did the photovoice study findings promote a social justice awareness, amelioration or transformation impact? We searched the PubMed, Scopus, PsycINFO and Web of Science databases from the years 2008-13, using the terms photonovella and photovoice and the combination of photovoice and social justice; and photovoice and participatory action research.

The original searches from all the databases combined yielded 695 articles. After all the duplicates were removed, articles were excluded if they met several criteria. They were excluded if (i) not written in the English language, (ii) not available in full text, (iii) published outside of 2008-13 (building upon Catalani and Minkler's review, which included articles that were published before 2008; $n=28$ ); (iv) were multiple reports from one research study $(n=3)$. Articles from the same study were reviewed initially and those studies did not add new information related to the purpose of this article. Thus, only the main article from the study which related specifically to photovoice and social justice was reviewed in this literature search. We also excluded references that $(\mathrm{v})$ were exploratory studies that utilized the photovoice method and were more focused on individual experiences and cultural approaches $(n=13)$; (vi) only discussed effectiveness/evaluation of the photovoice method with respect to the research study and did not provide a full study description $(n=16)$; and (vii) did not report photovoice findings to an audience $(n=29)$. Articles with these aforementioned concerns used photovoice merely as an additional source of data collection. For example, photovoice was used to triangulate data or used as a way to show pictures to emphasize research points. Consequently, a total of 30 research studies were reviewed (see Table 1).

\section{Analysis}

For analysis, a protocol was developed encompassing several steps to address our main study questions (Ganong 1987; Corbin and Strauss 1990). First, sections of the reports were highlighted to note the study approaches, including research purpose, guided methodology, study design, participant characteristics, approach to the photovoice method, dissemination of study findings (for example, the photo-exhibits whether via schools, forums, etc.) and the type of audience that attended those exhibitions. Second, each article was systematically reviewed where the texts were again revisited with specific focus on the study-findings sections and discussion to identify and match the reported social justice impacts with the social justice analysis framework we described earlier (e.g. whether a policy was developed and/or revised as a result of the study). Third, specific quotes were highlighted and recorded to note if the impact was more related to awareness, amelioration or transformation. Fourth, a matrix table was created and completed to compare the findings addressing our exploration during steps 1 and 2 (See Table 1). Fifth, using the table, the first two authors conferred about the noted impact and the implications of these summaries on the use and potential of the photovoice method.

\section{RESULTS}

\section{Methodology - Method Fit To Promote Social Justice}

Only a fraction of the studies (7/30) explicitly addressed social justice or inequity. (Halifax et al. 2008; Chilton et al. 2009; Duffy 2010; Foster-Fishman et al. 2010; Tanjasiri et al. 2011; Harper 2012; Markus 2012) For example, throughout their study report, Halifax et al. (2008) referenced to their underlying goal of promoting social justice. Their goal was to explore the social factors influencing the experiences of homeless individuals in Toronto. Markus (2012) used storytelling for social justice model-with the theoretical underpinnings in the critical race theory (CRT) - for data analysis.

Most authors (23/30) described their participants as vulnerable populations. When describing the photovoice 
Table 1 Addressing social justice through photovoice: methodology-method fit and social justice impacts

\begin{tabular}{|c|c|c|c|c|c|c|c|}
\hline No. & $\begin{array}{l}\text { Author }(s) \& c \\
\text { year }\end{array}$ & Purpose & Methodology & Participants & Exhibits \& audience & Direct impacts & $\begin{array}{l}\text { Aspects of } \\
\text { social justice } \\
\text { addressed } \\
\text { (awareness, } \\
\text { ameliorative or } \\
\text { transformative } \\
\text { impacts) }\end{array}$ \\
\hline 1 & $\begin{array}{l}\text { Andonian } \\
\text { and } \\
\text { MacRae } \\
(2011)\end{array}$ & $\begin{array}{l}\text { To explore social } \\
\text { participation } \\
\text { amongst older } \\
\text { adults whilst } \\
\text { living alone }\end{array}$ & $\begin{array}{l}\text { Mentioned } \\
\text { Action } \\
\text { research when } \\
\text { describing } \\
\text { photovoice, } \\
\text { but did not } \\
\text { use } \\
\text { participatory } \\
\text { action } \\
\text { approach }\end{array}$ & $\begin{array}{l}n=7 \text { urban older } \\
\text { adults who live } \\
\left.\text { alone (age } 55^{+}\right) \text {. } \\
\text { San Francisco, CA, } \\
\text { USA }\end{array}$ & $\begin{array}{l}\text { Exhibit: City hall: } \\
\text { Legislators, providers, } \\
\text { educators and others }\end{array}$ & Not reported & $\begin{array}{l}\text { Awareness } \\
\text { impact }\end{array}$ \\
\hline 2 & $\begin{array}{l}\text { Bharmal } \\
\text { et al. } \\
\text { (2012) }\end{array}$ & $\begin{array}{l}\text { To identify and } \\
\text { prioritize factors } \\
\text { associated with } \\
\text { the transition to } \\
\text { manhood } \\
\text { amongst African } \\
\text { American Men }\end{array}$ & $\begin{array}{l}\text { community- } \\
\text { based } \\
\text { participatory } \\
\text { research } \\
\text { (CBPR) - to } \\
\text { create } \\
\text { partnerships }\end{array}$ & $\begin{array}{l}n=12 \text { African- } \\
\text { American men } \\
\text { (ages 16-26 years). } \\
\text { Los Angeles, CA, } \\
\text { USA }\end{array}$ & $\begin{array}{l}\text { Exhibit: Community } \\
\text { forums: secondary } \\
\text { school members. }\end{array}$ & $\begin{array}{l}\text { Designed and } \\
\text { implemented a young } \\
\text { men's access support } \\
\text { group }\end{array}$ & $\begin{array}{l}\text { Awareness \& } \\
\text { ameliorative } \\
\text { impacts }\end{array}$ \\
\hline 3 & $\begin{array}{l}\text { Brazg et al. } \\
\quad(2011)\end{array}$ & $\begin{array}{l}\text { To assess } \\
\text { adolescent } \\
\text { substance use } \\
\text { and abuse. }\end{array}$ & $\begin{array}{l}\text { CBPR - to } \\
\text { create } \\
\text { partnerships }\end{array}$ & $\begin{array}{l}n=12 \text { high school } \\
\text { students (grades } \\
10,11,12 \text { ) WA, USA }\end{array}$ & $\begin{array}{l}\text { Exhibit: Community } \\
\text { forum: alcohol and } \\
\text { drug intervention } \\
\text { specialists, youth and } \\
\text { family services } \\
\text { director, city attorney, } \\
\text { and local police }\end{array}$ & Not reported & $\begin{array}{l}\text { Awareness } \\
\text { impact }\end{array}$ \\
\hline 4 & $\begin{array}{l}\text { Castleden } \\
\text { et al. } \\
(2008)\end{array}$ & $\begin{array}{l}\text { To evaluate the } \\
\text { use of } \\
\text { photovoice }\end{array}$ & $\begin{array}{l}\text { CBPR - } \\
\text { Complete }\end{array}$ & $\begin{array}{l}n=45 \text { (ages } 19- \\
75 \text { years) First } \\
\text { Nation in Western } \\
\text { Canada }\end{array}$ & $\begin{array}{l}\text { Exhibit: Newsletters } \\
\text { and posters during } \\
\text { potluck dinners at } \\
\text { four community sites } \\
\text { within the community } \\
\text { and upon request. }\end{array}$ & $\begin{array}{l}\text { Two community } \\
\text { members trained in } \\
\text { photovoice research } \\
\text { and are using it to } \\
\text { address other } \\
\text { environmental and } \\
\text { health issues }\end{array}$ & $\begin{array}{l}\text { Awareness \& } \\
\text { ameliorative } \\
\text { impacts }\end{array}$ \\
\hline 5 & $\begin{array}{l}\text { Chilton et al. } \\
\text { (2009) }\end{array}$ & $\begin{array}{l}\text { To record single } \\
\text { mothers' stories } \\
\text { on poverty and } \\
\text { hunger to } \\
\text { inform social } \\
\text { welfare policy }\end{array}$ & $\begin{array}{l}\text { Human rights } \\
\text { framework. } \\
\text { Participatory } \\
\text { advocacy }\end{array}$ & $\begin{array}{l}n=42 \text { mothers of } \\
\text { young children }\end{array}$ & $\begin{array}{l}\text { Exhibit: (i) Interactive } \\
\text { searchable and socially } \\
\text { networked website, } \\
\text { (ii) United States } \\
\text { Senate by } 3 \text { US } \\
\text { senators, (iii) } \\
\text { Congressman Jim } \\
\text { McGovern home } \\
\text { district in } \\
\text { Massachusetts, (iv) } \\
\text { senate democratic } \\
\text { steering and outreach } \\
\text { committee }\end{array}$ & $\begin{array}{l}\text { Women shared stories } \\
\text { and images through } \\
\text { internet, and public } \\
\text { forums and media. }\end{array}$ & $\begin{array}{l}\text { Awareness } \\
\text { impact }\end{array}$ \\
\hline 6 & $\begin{array}{l}\text { Davison, } \\
\text { Ghali, and } \\
\text { Hawe } \\
\text { (2011) }\end{array}$ & $\begin{array}{l}\text { To Determine } \\
\text { interventions to } \\
\text { foster social } \\
\text { inclusion and } \\
\text { improve student } \\
\text { health }\end{array}$ & $\begin{array}{l}\text { Mentioned } \\
\text { participatory } \\
\text { action } \\
\text { research whilst } \\
\text { describing } \\
\text { photovoice } \\
\text { but did not } \\
\text { use that } \\
\text { approach. }\end{array}$ & $\begin{array}{l}n=10 \text { young } \\
\text { students }\end{array}$ & $\begin{array}{l}\text { Exhibit: School display: } \\
\text { Students and staff }\end{array}$ & $\begin{array}{l}\text { As a result of the study, } \\
\text { teachers consciously } \\
\text { worked to prevent } \\
\text { harassment of } \\
\text { younger students, } \\
\text { instituted new } \\
\text { measures for ESL } \\
\text { students and a formal } \\
\text { orientation. }\end{array}$ & $\begin{array}{l}\text { Awareness and } \\
\text { ameliorative } \\
\text { impacts }\end{array}$ \\
\hline
\end{tabular}


Table 1 Continued

\begin{tabular}{|c|c|c|c|c|c|c|c|}
\hline No. & $\begin{array}{l}\text { Author }(\mathrm{s}) \& \\
\text { year }\end{array}$ & Purpose & Methodology & Participants & Exhibits \& audience & Direct impacts & $\begin{array}{l}\text { Aspects of } \\
\text { social justice } \\
\text { addressed } \\
\text { (awareness, } \\
\text { ameliorative or } \\
\text { transformative } \\
\text { impacts) }\end{array}$ \\
\hline 7 & $\begin{array}{l}\text { Denov et al. } \\
(2012)\end{array}$ & $\begin{array}{l}\text { To Explore the } \\
\text { post war \& } \\
\text { reintegration } \\
\text { experiences of } \\
\text { former child } \\
\text { soldiers }\end{array}$ & $\begin{array}{l}\text { CBPR - to } \\
\text { create } \\
\text { partnerships } \\
\text { with the } \\
\text { project. }\end{array}$ & $\begin{array}{l}n=11 \text { Youth (ages } \\
\text { 18-23 years) Bedelu } \\
\text { slum, Freetown, } \\
\text { Sierra Leone }\end{array}$ & $\begin{array}{l}\text { Exhibit: Community } \\
\text { forums: government, } \\
\text { local, and } \\
\text { international NGOs, } \\
\text { youths and local } \\
\text { media. Exhibited at } \\
\text { McGill University in } \\
\text { Canada: study } \\
\text { participants, faculty, } \\
\text { students and the } \\
\text { public. }\end{array}$ & Not reported & $\begin{array}{l}\text { Awareness } \\
\text { impact }\end{array}$ \\
\hline 8 & $\begin{array}{l}\text { Downey, } \\
\text { Ireson, and } \\
\text { Scutchfield } \\
\text { 2009) }\end{array}$ & $\begin{array}{l}\text { To explore the } \\
\text { empowerment } \\
\text { education model } \\
\text { and community } \\
\text { health } \\
\text { assessment }\end{array}$ & $\begin{array}{l}\text { participatory } \\
\text { action } \\
\text { research } \\
(\mathrm{PAR})\end{array}$ & $\begin{array}{l}n=18 \text { (ages } 15- \\
18 \text { years). rural } \\
\text { citizens of one } \\
\text { Appalachian } \\
\text { county, USA }\end{array}$ & $\begin{array}{l}\text { Exhibit: Community } \\
\text { forum at local diner, } \\
\text { community centre, } \\
\text { high school, and a } \\
\text { church: community } \\
\text { leaders and } \\
\text { stakeholders }\end{array}$ & Not reported & $\begin{array}{l}\text { Awareness } \\
\text { impact }\end{array}$ \\
\hline 9 & Duffy (2010) & $\begin{array}{l}\text { To record, reflect } \\
\text { and act on } \\
\text { community } \\
\text { influences on } \\
\text { health; to } \\
\text { promote critical } \\
\text { dialogue and to } \\
\text { reach policy } \\
\text { makers. }\end{array}$ & PAR & $\begin{array}{l}n=7 \text { (ages } 18+\text { ) } \\
\text { single mothers in } \\
\text { Moncton, New } \\
\text { Brunswick, Canada }\end{array}$ & $\begin{array}{l}\text { Exhibits: partner } \\
\text { agency board; an } \\
\text { open house; a three- } \\
\text { day event: Mayor and } \\
\text { media; Various public } \\
\text { venues and } \\
\text { conferences; radio, } \\
\text { newspaper, and } \\
\text { television (regional } \\
\text { and national) } \\
\text { coverage. }\end{array}$ & $\begin{array}{l}\text { The women } \\
\text { implemented: (i) an } \\
\text { survey of public } \\
\text { transit, (ii) A white } \\
\text { paper for accessible } \\
\text { public transport } \\
\text { presented to: } \\
\text { (a) the general } \\
\text { manager Transit } \\
\text { System, (b) transit } \\
\text { board, (iii) other } \\
\text { evaluations of transit }\end{array}$ & $\begin{array}{l}\text { Awareness \& } \\
\text { ameliorative } \\
\text { impacts }\end{array}$ \\
\hline 10 & $\begin{array}{l}\text { Findholt } \\
\text { et al. } \\
\text { (2011) }\end{array}$ & $\begin{array}{l}\text { To assess for } \\
\text { obesity } \\
\text { prevention and } \\
\text { physical activity. }\end{array}$ & $\begin{array}{l}\text { Mentioned } \\
\text { CBPR as they } \\
\text { were } \\
\text { describing } \\
\text { photovoice }\end{array}$ & $\begin{array}{l}n=6 \text { (ages } 15- \\
18 \text { years })\end{array}$ & $\begin{array}{l}\text { Exhibit: high school } \\
\text { auditorium: coalition } \\
\text { members, parents, } \\
\text { teachers, school } \\
\text { administrators, city } \\
\text { and county } \\
\text { government } \\
\text { employees, health } \\
\text { care professionals, } \\
\text { business leaders, and } \\
\text { local media. }\end{array}$ & Not reported & Awareness \\
\hline 11 & $\begin{array}{l}\text { Foster- } \\
\text { Fishman } \\
\text { et al. } \\
(2010)\end{array}$ & $\begin{array}{l}\text { To learn about } \\
\text { young people } \\
\text { involvement, } \\
\text { support in } \\
\text { neighbourhoods, } \\
\text { schools and } \\
\text { community }\end{array}$ & $\begin{array}{l}\text { PAR - involved } \\
\text { participants in } \\
\text { all phases of } \\
\text { the research. }\end{array}$ & $\begin{array}{l}n=19 \text { middle school } \\
\text { students } \\
\text { (representing four } \\
\text { middle schools; ages } \\
\text { 12-13) }\end{array}$ & $\begin{array}{l}\text { Exhibit: Retreat for } \\
\text { local organizations } \\
\text { and residents }\end{array}$ & $\begin{array}{l}\text { Published guide about } \\
\text { youth concerns. As a } \\
\text { result, youths' } \\
\text { concern was the focal } \\
\text { point during two } \\
\text { major community } \\
\text { events }\end{array}$ & $\begin{array}{l}\text { Awareness \& } \\
\text { ameliorative } \\
\text { impacts }\end{array}$ \\
\hline
\end{tabular}


Table 1 Continued

\begin{tabular}{|c|c|c|c|c|c|c|c|}
\hline No. & $\begin{array}{l}\text { Author }(\mathrm{s}) \& \\
\text { year }\end{array}$ & Purpose & Methodology & Participants & Exhibits \& audience & Direct impacts & $\begin{array}{l}\text { Aspects of } \\
\text { social justice } \\
\text { addressed } \\
\text { (awareness, } \\
\text { ameliorative or } \\
\text { transformative } \\
\text { impacts) }\end{array}$ \\
\hline 12 & $\begin{array}{l}\text { Flum et al. } \\
(2010)\end{array}$ & $\begin{array}{l}\text { To Explore } \\
\text { hazards for } \\
\text { workplace injury }\end{array}$ & $\begin{array}{l}\text { Mentioned } \\
\text { PAR when } \\
\text { describing } \\
\text { photovoice }\end{array}$ & $\begin{array}{l}n=16 \text { University } \\
\text { Custodians }\end{array}$ & $\begin{array}{l}\text { Exhibit: Forum: } \\
\text { custodians and } \\
\text { occupational safety } \\
\text { and health } \\
\text { stakeholders }\end{array}$ & $\begin{array}{l}\text { Improved waste } \\
\text { removal; } \\
\text { ergonomically safer } \\
\text { equipment } \\
\text { purchased; } \\
\text { management } \\
\text { disseminated exhibit } \\
\text { via website. } \\
\text { Established safety } \\
\text { committee for } \\
\text { custodians has been } \\
\text { established; and } \\
\text { custodian injuries had } \\
\text { decreased from } 39 \% \\
\text { in } 2007 \text { (year of the } \\
\text { study) to } 26 \% \text { in } 2008 \\
\text { and } 20 \% \text { in } 2009\end{array}$ & $\begin{array}{l}\text { Awareness \& } \\
\text { ameliorative } \\
\text { impacts }\end{array}$ \\
\hline 13 & $\begin{array}{l}\text { Grieb et al. } \\
(2013)\end{array}$ & $\begin{array}{l}\text { Housing residents } \\
\text { perspectives on } \\
\text { housing and } \\
\text { health }\end{array}$ & $\begin{array}{l}\text { Used CBPR } \\
\text { when } \\
\text { describing } \\
\text { photovoice }\end{array}$ & $\begin{array}{l}n=9 \text { men in } \\
\text { transitional } \\
\text { housing, returning } \\
\text { from incarceration, } \\
\text { Baltimore }\end{array}$ & $\begin{array}{l}\text { Exhibit: community } \\
\text { based organizations, } \\
\text { two libraries, and city } \\
\text { hall }\end{array}$ & $\begin{array}{l}\text { Community and } \\
\text { academic partnership } \\
\text { for community clean } \\
\text { up projects and for } \\
\text { development of a } \\
\text { family intervention } \\
\text { programme }\end{array}$ & $\begin{array}{l}\text { Awareness \& } \\
\text { ameliorative }\end{array}$ \\
\hline 14 & $\begin{array}{l}\text { Green and } \\
\text { Kloos } \\
(2009)\end{array}$ & $\begin{array}{l}\text { To document } \\
\text { youths' life in } \\
\text { community }\end{array}$ & $\begin{array}{l}\text { Mentioned } \\
\text { PAR when } \\
\text { describing } \\
\text { photovoice }\end{array}$ & $\begin{array}{l}n=12 \text { migrant } \\
\text { youths from } \\
\text { Uganda (ages } \\
12-16 \text { years) }\end{array}$ & $\begin{array}{l}\text { Exhibit: website; four } \\
\text { small cafes in North } \\
\text { Carolina and South } \\
\text { Carolina (USA) }\end{array}$ & $\begin{array}{l}\text { Fundraising for: laptop } \\
\text { computers }(n=2) \\
\text { and school fees for } \\
\text { participants }\end{array}$ & $\begin{array}{l}\text { Awareness \& } \\
\text { ameliorative } \\
\text { impacts }\end{array}$ \\
\hline 15 & $\begin{array}{l}\text { Halifax et al. } \\
(2008)\end{array}$ & $\begin{array}{l}\text { To document and } \\
\text { make changes in } \\
\text { the homelessness } \\
\text { community. }\end{array}$ & CBPR & $\begin{array}{l}n=12 \text { homeless } \\
\text { women and men } \\
\text { (ages } 20-60 \text { ) } \\
\text { Toronto, Canada }\end{array}$ & $\begin{array}{l}\text { Exhibit: informal and } \\
\text { formal meetings with } \\
\text { politicians, } \\
\text { community events, } \\
\text { written reports and } \\
\text { publications. }\end{array}$ & $\begin{array}{l}\text { Increased involvement } \\
\text { of homeless people in } \\
\text { the community }\end{array}$ & $\begin{array}{l}\text { Awareness } \\
\text { impact }\end{array}$ \\
\hline 16 & $\begin{array}{l}\text { Hannay et al. } \\
\text { (2013) }\end{array}$ & $\begin{array}{l}\text { To identify } \\
\text { barriers to } \\
\text { physical activity } \\
\text { and initiating } \\
\text { policy-change } \\
\text { actions }\end{array}$ & $\begin{array}{l}\text { Mentioned } \\
\text { CBPR when } \\
\text { describing } \\
\text { photovoice }\end{array}$ & $\begin{array}{l}\text { Six adults and } 19 \\
\text { teens }\end{array}$ & $\begin{array}{l}\text { Presentation of photos } \\
\text { and reflections were } \\
\text { made at the } \\
\text { Connecticut's 2nd } \\
\text { Annual Physical } \\
\text { Activity and Nutrition } \\
\text { Symposium. The } \\
\text { audience included } \\
\text { health providers, } \\
\text { educators, } \\
\text { policymakers and } \\
\text { community members }\end{array}$ & & Awareness \\
\hline 17 & $\begin{array}{l}\text { Haque and } \\
\text { Eng (2011) }\end{array}$ & $\begin{array}{l}\text { To Record } \\
\text { neighbourhood } \\
\text { implications on } \\
\text { residents' health }\end{array}$ & $\begin{array}{l}\text { Community- } \\
\text { based research } \\
(\mathrm{CBR})\end{array}$ & $\begin{array}{l}n=27 \text { (ages } \\
18-68 \text { years) } \\
\text { St. Jamestown } \\
\text { Canada }\end{array}$ & $\begin{array}{l}\text { Exhibit: community } \\
\text { forum ( } n=300) \text { and } \\
\text { City Hall: city } \\
\text { councillor, and } 15 \text { self- } \\
\text { invited municipal } \\
\text { representatives }\end{array}$ & $\begin{array}{l}\text { Conducted an } \\
\text { inventory/ } \\
\text { replacement/repair } \\
\text { of neighbourhood } \\
\text { bicycle racks }\end{array}$ & $\begin{array}{l}\text { Awareness \& } \\
\text { ameliorative } \\
\text { impacts }\end{array}$ \\
\hline
\end{tabular}


Table 1 Continued

\begin{tabular}{|c|c|c|c|c|c|c|c|}
\hline No. & $\begin{array}{l}\text { Author }(\mathrm{s}) \& \\
\text { year }\end{array}$ & Purpose & Methodology & Participants & Exhibits \& audience & Direct impacts & $\begin{array}{l}\text { Aspects of } \\
\text { social justice } \\
\text { addressed } \\
\text { (awareness, } \\
\text { ameliorative or } \\
\text { transformative } \\
\text { impacts) }\end{array}$ \\
\hline 18 & $\begin{array}{r}\text { Harper } \\
(2012)\end{array}$ & $\begin{array}{l}\text { To document } \\
\text { environmental } \\
\text { issues }\end{array}$ & $\begin{array}{l}\text { PAR - actually } \\
\text { had the } \\
\text { community } \\
\text { leaders } \\
\text { involved in the } \\
\text { research } \\
\text { process }\end{array}$ & $\begin{array}{c}n=6 \text { Romani Adults } \\
\text { (ages 18-24 years) } \\
\text { Northern Hungary }\end{array}$ & $\begin{array}{l}\text { Exhibit: Sajoszentpeter } \\
\text { \& Budapest: } \\
\text { community members. } \\
\text { The mayor, local } \\
\text { council members, } \\
\text { public administrators, } \\
\text { doctors, nurses, } \\
\text { teachers, } \\
\text { environmental } \\
\text { NGO's, media, } \\
\text { activists and lawyers. } \\
\text { UN committees } \\
(n=2)\end{array}$ & $\begin{array}{l}\text { Developed: (i) } \\
\text { proposal for a } \\
\text { nationwide project } \\
\text { addressing social } \\
\text { justice and } \\
\text { environmental issues, } \\
\text { (ii) Policy } \\
\text { recommendations on } \\
\text { environmental justice. }\end{array}$ & $\begin{array}{l}\text { Awareness \& } \\
\text { ameliorative } \\
\text { impacts }\end{array}$ \\
\hline 19 & $\begin{array}{l}\text { Kramer et al. } \\
(2010)\end{array}$ & $\begin{array}{l}\text { To enable policy } \\
\text { makers to be } \\
\text { actively involved } \\
\text { in identifying } \\
\text { community } \\
\text { needs }\end{array}$ & CBPR & $\begin{array}{l}n=44(29 \text { adults and } \\
15 \text { youth }) \text { from } \\
\text { Kaiser Permanente } \\
\text { Colorado, USA }\end{array}$ & $\begin{array}{l}\text { Exhibit: invitation-only } \\
\text { policy roundtable, an } \\
\text { active living summit } \\
\text { local festivals, } \\
\text { business, community } \\
\text { centres, churches and } \\
\text { planning department } \\
\text { meetings: } \\
\text { Policymakers, State } \\
\text { Governor, \& media }\end{array}$ & $\begin{array}{l}\text { Voter initiative passed } \\
\text { to renovate the City } \\
\text { Park. Full service } \\
\text { grocery store, } \\
\text { watershed project and } \\
\text { walkable trails built }\end{array}$ & $\begin{array}{l}\text { Awareness, } \\
\text { ameliorative, } \\
\text { and } \\
\text { transformative } \\
\text { impacts }\end{array}$ \\
\hline 20 & $\begin{array}{l}\text { Lardeau, } \\
\text { Healey, and } \\
\text { Ford (2011) }\end{array}$ & $\begin{array}{l}\text { To explore } \\
\text { determinants of } \\
\text { food insecurity } \\
\text { amongst food } \\
\text { programme } \\
\text { users }\end{array}$ & $\begin{array}{l}\text { Mentioned } \\
\text { CBPR when } \\
\text { describing } \\
\text { photovoice }\end{array}$ & $\begin{array}{l}n=8 \text { regular users of } \\
\text { food programmes }\end{array}$ & $\begin{array}{l}\text { Exhibit: Museum. No } \\
\text { reports of attendance. }\end{array}$ & Not reported & $\begin{array}{l}\text { Awareness } \\
\text { impact }\end{array}$ \\
\hline 21 & $\begin{array}{l}\text { Lorenz and } \\
\text { Kolb (2009) }\end{array}$ & $\begin{array}{l}\text { To understand } \\
\text { and raise } \\
\text { awareness about } \\
\text { brain injury }\end{array}$ & $\begin{array}{l}\text { CBPR - } \\
\text { Actually } \\
\text { involved } \\
\text { participants in } \\
\text { the research } \\
\text { process (e.g. } \\
\text { recruitment) }\end{array}$ & $\begin{array}{l}n=8 \text { individuals } \\
\text { from the brain } \\
\text { injury survivor } \\
\text { support group. MA, } \\
\text { USA }\end{array}$ & $\begin{array}{l}\text { Exhibit: Three libraries, } \\
\text { two brain injury } \\
\text { conferences, a } \\
\text { neurology conference, } \\
\text { the Massachusetts } \\
\text { State house and the } \\
\text { Brain injury } \\
\text { Association of } \\
\text { Massachusetts }\end{array}$ & $\begin{array}{l}\text { Social networking } \\
\text { website. Public library } \\
\text { to raise awareness } \\
\text { about the problem }\end{array}$ & $\begin{array}{l}\text { Awareness } \\
\text { impact }\end{array}$ \\
\hline 22 & $\begin{array}{r}\text { Markus } \\
(2012)\end{array}$ & $\begin{array}{l}\text { To empower } \\
\text { healthy } \\
\text { relationships \& } \\
\text { prevention of } \\
\text { HIV unintended } \\
\text { pregnancy }\end{array}$ & $\begin{array}{l}\text { Used PAR } \\
\text { (discussed the } \\
\text { social justice } \\
\text { framework) }\end{array}$ & $\begin{array}{l}n=6 \text { (18-19 years) } \\
\text { American Indian } \\
\text { Wind River, WY, } \\
\text { USA }\end{array}$ & $\begin{array}{l}\text { Exhibit: University } \\
\text { symposium on social } \\
\text { justice and an art } \\
\text { reception: parents, } \\
\text { grandparents, Tribal } \\
\text { leaders, college } \\
\text { counsellors, teachers, } \\
\text { youths and } \\
\text { community mental } \\
\text { health providers. }\end{array}$ & Peer education plan & $\begin{array}{l}\text { Awareness } \\
\text { impact }\end{array}$ \\
\hline
\end{tabular}


Table 1 Continued

\begin{tabular}{|c|c|c|c|c|c|c|c|}
\hline No. & $\begin{array}{l}\text { Author }(\mathrm{s}) \& \mathrm{c} \\
\text { year }\end{array}$ & Purpose & Methodology & Participants & Exhibits \& audience & Direct impacts & $\begin{array}{l}\text { Aspects of } \\
\text { social justice } \\
\text { addressed } \\
\text { (awareness, } \\
\text { ameliorative or } \\
\text { transformative } \\
\text { impacts) }\end{array}$ \\
\hline 23 & $\begin{array}{l}\text { Mohammed, } \\
\text { Sajun, and } \\
\text { Khan } \\
\text { (2013) }\end{array}$ & $\begin{array}{l}\text { To explore the } \\
\text { experiences of } \\
\text { people with } \\
\text { Tuberculosis and } \\
\text { to advocate for a } \\
\text { supportive } \\
\text { environment for } \\
\text { them }\end{array}$ & Not reported & $\begin{array}{l}\text { Individuals ( } 15 \text { years } \\
\text { and older) who } \\
\text { were directly (e.g. } \\
\text { tuberculosis } \\
\text { patients) and } \\
\text { indirectly (e.g. } \\
\text { family members) } \\
\text { affected by } \\
\text { tuberculosis. }\end{array}$ & $\begin{array}{l}\text { A call for action was } \\
\text { developed and } \\
\text { presented a gallery } \\
\text { event. The call for } \\
\text { action included six } \\
\text { steps to reduce the } \\
\text { spread of tuberculosis } \\
\text { and having a } \\
\text { supporting } \\
\text { environment. About } \\
1000 \text { people saw the } \\
\text { gallery, which was a } \\
\text { three day display. Also } \\
\text { seven news channels } \\
\text { (32 minutes to one } \\
\text { hour air time), } \\
\text { newspapers and other } \\
\text { online sources } \\
\text { covered the event. }\end{array}$ & Not reported & Awareness \\
\hline 24 & $\begin{array}{l}\text { Newman } \\
(2010)\end{array}$ & $\begin{array}{l}\text { To record the } \\
\text { environmental } \\
\text { barriers and } \\
\text { facilitators to } \\
\text { community } \\
\text { participation }\end{array}$ & $\begin{array}{l}\text { CBPR - actually } \\
\text { built } \\
\text { relationships } \\
\text { with } \\
\text { community } \\
\text { members } \\
\text { prior to the } \\
\text { study }\end{array}$ & $\begin{array}{l}n=10 \text { adults with } \\
\text { spinal cord injury } \\
\text { Charleston, SC, } \\
\text { USA }\end{array}$ & $\begin{array}{l}\text { Exhibit: Written } \\
\text { testimony: South } \\
\text { Carolina Senate } \\
\text { Transportation } \\
\text { Committee. } \\
\text { Newspaper published } \\
\text { stories }\end{array}$ & $\begin{array}{l}\text { Coalition developed. } \\
\text { State law to } \\
\text { strengthen accessible } \\
\text { parking laws } \\
\text { proposed. }\end{array}$ & $\begin{array}{l}\text { Awareness and } \\
\text { transformative } \\
\text { impacts }\end{array}$ \\
\hline 25 & $\begin{array}{l}\text { Pritzker, } \\
\text { LaChapelle, } \\
\text { and Tatum } \\
(2012)\end{array}$ & $\begin{array}{l}\text { To empower } \\
\text { participation in } \\
\text { civic engagement }\end{array}$ & $\begin{array}{l}\text { CBPR - but } \\
\text { participants } \\
\text { not involved } \\
\text { in the process }\end{array}$ & $\begin{array}{l}n=15 \text { high school } \\
\text { students }\end{array}$ & $\begin{array}{l}\text { Exhibit: School } \\
\text { campus: parents, } \\
\text { university professors, } \\
\text { teachers, city and state } \\
\text { policymakers }\end{array}$ & Not reported & $\begin{array}{l}\text { Awareness } \\
\text { impact }\end{array}$ \\
\hline 26 & $\begin{array}{l}\text { Poudrier and } \\
\text { Mac-Lean } \\
(2009) \\
\text { Awareness } \\
\text { impact }\end{array}$ & $\begin{array}{l}\text { To explore and } \\
\text { make visible } \\
\text { experiences with } \\
\text { breast cancer }\end{array}$ & Decolonizing & $\begin{array}{l}\text { Methodologies } \\
\text { (Feminist } \\
\text { Epistemology) }\end{array}$ & $\begin{array}{l}n=12 \text { (ages } 42-75) \\
\text { Aboriginal women, } \\
\text { Saskatchewan, Canada }\end{array}$ & $\begin{array}{l}\text { Exhibit: Community } \\
\text { forum: key } \\
\text { stakeholders, } \\
\text { advocacy groups }\end{array}$ & Not reported \\
\hline 27 & $\begin{array}{l}\text { Seitz et al. } \\
(2012)\end{array}$ & $\begin{array}{l}\text { To empower } \\
\text { students to } \\
\text { advocate for } \\
\text { change of a } \\
\text { campus smoking } \\
\text { policy }\end{array}$ & $\begin{array}{l}\text { Mentioned } \\
\text { CBPR when } \\
\text { describing the } \\
\text { photovoice } \\
\text { method }\end{array}$ & $\begin{array}{l}n=49 \\
\text { undergraduate } \\
\text { college students } \\
\text { STATE, USA }\end{array}$ & $\begin{array}{l}\text { Exhibit: University's } \\
\text { student union } \\
\text { building: faculty, staff, } \\
\text { students and } \\
\text { community members }\end{array}$ & $\begin{array}{l}\text { Ashtrays were } \\
\text { relocated/removed by } \\
\text { the university's } \\
\text { Grounds Crew } \\
\text { Supervisor. Students } \\
\text { planned to write a } \\
\text { version of an ideal } \\
\text { policy. }\end{array}$ & $\begin{array}{l}\text { Awareness \& } \\
\text { ameliorative } \\
\text { impacts }\end{array}$ \\
\hline 28 & $\begin{array}{l}\text { Tanjasiri } \\
\text { et al. } \\
(2011)\end{array}$ & $\begin{array}{l}\text { To identify and } \\
\text { understand } \\
\text { environmental } \\
\text { characteristics } \\
\text { associated with } \\
\text { tobacco use }\end{array}$ & $\begin{array}{l}\text { CBPR - } \\
\text { Community } \\
\text { leaders were } \\
\text { involved in the } \\
\text { process }\end{array}$ & $\begin{array}{l}n=32 \text { youths ages } \\
14-18 \text { years WA \& } \\
\text { CA, USA }\end{array}$ & $\begin{array}{l}\text { Exhibit: meeting of } \\
\text { tobacco control } \\
\text { advocates, \& City } \\
\text { Council. }\end{array}$ & $\begin{array}{l}\text { The proposal that the } \\
\text { students supported } \\
\text { was passed and } \\
\text { enacted in } 2008 \text {. }\end{array}$ & $\begin{array}{l}\text { Awareness \& } \\
\text { transformative } \\
\text { impacts }\end{array}$ \\
\hline
\end{tabular}


Table 1 Continued

\begin{tabular}{|c|c|c|c|c|c|c|c|}
\hline No. & $\begin{array}{l}\text { Author }(\mathrm{s}) \& \mathrm{c} \\
\text { year }\end{array}$ & Purpose & Methodology & Participants & Exhibits \& audience & Direct impacts & $\begin{array}{l}\text { Aspects of } \\
\text { social justice } \\
\text { addressed } \\
\text { (awareness, } \\
\text { ameliorative or } \\
\text { transformative } \\
\text { impacts) }\end{array}$ \\
\hline 29 & $\begin{array}{l}\text { Valera et al. } \\
\text { (2009) }\end{array}$ & $\begin{array}{l}\text { To document } \\
\text { challenges } \\
\text { accessing healthy } \\
\text { food in }\end{array}$ & $\begin{array}{l}\text { Mentioned } \\
\text { PAR during } \\
\text { photovoice } \\
\text { description }\end{array}$ & $\begin{array}{l}n=9 \text { low income } \\
\text { women (ages 20- } \\
45) \text { New York City, } \\
\text { NY, USA }\end{array}$ & $\begin{array}{l}\text { Exhibit: College } \\
\text { conference, \& in } \\
\text { Letters to } \\
\text { policymakers }\end{array}$ & Not reported & $\begin{array}{l}\text { Awareness } \\
\text { impact }\end{array}$ \\
\hline 30 & $\begin{array}{l}\text { Vaughn } \\
\text { et al. } \\
\text { (2009) }\end{array}$ & $\begin{array}{l}\text { To evaluate home } \\
\text { visitation } \\
\text { programme }\end{array}$ & PAR & $\begin{array}{l}n=7 \text { mothers } \\
\text { Cincinnati, KY, USA }\end{array}$ & $\begin{array}{l}\text { Exhibit: Community } \\
\text { forum: mothers, } \\
\text { home visitors, policy } \\
\text { makers and } \\
\text { programme service } \\
\text { providers. }\end{array}$ & Not Reported & $\begin{array}{l}\text { Awareness } \\
\text { impact }\end{array}$ \\
\hline
\end{tabular}

method, they also discussed that the underlying intent of the method was to foster empowerment and promote change amongst those researched. However, they did not clearly discuss or identify the structural conditions (social oppressions and political conditions) constituting to participant vulnerability.

Almost half $(13 / 30)$ clearly identified their research methodology. Three were explicit about their methodological framework and most (10/30) used principles of participatory action research (PAR). One study (Poudrier and MacLean 2009) was guided by the decolonizing methodology and the feminist epistemology. Another study (Markus 2012) described using a social determinants of health and social ecological model. Chilton et al. (2009) used a human rights framework to guide their study.

Ten studies (Castleden, Garvin and First Nation 2008; Lorenz and Kolb 2009; Vaughn, Forbes and Howell 2009; Foster-Fishman et al. 2010; Newman 2010; Brazg et al. 2011; Tanjasiri et al. 2011; Bharmal et al. 2012; Denov, Doucet and Kamara 2012; Harper 2012; Grieb et al. 2013) used core principles of PAR involving participants in all aspects of the research process. For example, Foster-Fishman et al. (2010) utilized the ReACT method to involve their youth participants in data analysis leading to theme emergence.

The remaining studies only mentioned participatory research in terms of acknowledging the community-based participatory research (CBPR) origins of the photovoice method. Photovoice was used to promote participants' involvement in data gathering. However, the researchers enlisted support of community members with a pre-determined research question and pre-identified problem to be explored. It was not clear whether community members assisted in identifying community needs, formulated the research question or explored ways to proceed with the research process. In these studies, following the photovoice sessions, participants were also not involved in the data analysis process.

\section{Social Justice Impact: Awareness, Amelioration and Transformation}

Researchers from all 30 studies reported that they raised awareness amongst the participants, community members and targeted stakeholders. For example, one group stated:

Through their own discussions and by sharing similar stories with each other, the participants voiced frustration and anger about coming to terms with this awareness of their circumstances within the context of their community.

(Valera et al. 2009)

Another noted:

The method was very useful for increasing public awareness of the conditions in Union County that affect children's physical activity and diets.

(Findholt, Michael and Davis 2011)

These statements are exemplars of how the researchers reported awareness-raising. The former statement showed awareness-raising at the individual level, whilst the latter indicates awareness-raising at the community level. Two studies used direct quotes from participants to demonstrate increased awareness (Findholt et al. 2011; Hannay et al. 2013).

Eleven of the studies resulted in some form of immediate action to ameliorate the issue at hand. For example, Duffy 
(2010) reported that the participants were invited to review and evaluate the transit improvements. Based on participants' suggestions, changes regarding safety and scheduling were undertaken.

Only three of the studies prompted changes at the policy level for transformative impact. For example, the impact of Newman (2010) was the passage of a state law to strengthen accessible parking for handicaps. Kramer et al.' (2010)s study spurred a successful voter initiative to renovate the city park and build a full service grocery store, a watershed project and walkable trails. Finally, Tanjasiri et al. (2011) described the passing and enactment of a proposal that required licensing amongst tobacco vendors. These studies evidenced that photovoice research studies can lead to a social justice impact.

\section{DISCUSSION}

The purposes of this article were to explore (i) whether authors related the methodologies to their aims of promoting social justice (methodology-method fit) and (ii) outline the social justice research impact of photovoice findings using the framework of social justice awareness, amelioration and transformation. Our present review supports the assertion made by Catalani and Minkler (2010) that research designs involving photovoice continue to under-deliver their action potential.

Furthermore, this is the first review article that examined how researchers using the photovoice method describe methodology-method fit and social justice impact. This is important because the original concerns of photovoice were oriented towards community-level justice actions. This review documented that few studies are designed for this goal and instead accomplish change only at the individual level. To this end, there continues to be a lack of consistency amongst photovoice studies in identifying a guiding methodology. Many photovoice studies used community-based participatory action research and participatory-action approaches as a way of describing their social justice intent. However, there has been no concrete description of how participatory study designs using photovoice have addressed social justice. This lack of explicit detail possibly resulted from research and publishing expectations (Castleden et al. 2008) because of the pressure on the researchers to conform to publication requirements and academic standards of rigour. Awarenessraising as a result of using the photovoice method have been described elsewhere (Foster-Fishman et al. 2005; Carlson, Engebretson and Chamberlain 2006). The findings in this review suggest that there is a need for photovoice study design to reach beyond a focus on individual impact to focus on system-level impacts. Only three of the studies reviewed (Kramer et al. 2010; Newman 2010; Tanjasiri et al. 2011) described transformative change in material or political circumstances for participants and communities involved. Whilst studies clearly reported awareness impacts in participants, researchers and other audiences, there were not many reports on ameliorative or transformative social justice impacts.

The photovoice method lends itself to implicit assumptions about awareness-raising. The act of seeing and discussing photos is assumed to inspire awareness of new ways of thinking about inequity and health through the images produced by those disadvantaged. Limitations of these assumptions were not discussed by the researchers in the studies reviewed. Indeed, few researchers took the opportunity to adequately describe the importance of the awareness for themselves on the research enterprise or on reducing health inequity. None discussed the indirect impact of this awareness on the potential audiences for the published research for community-based dissemination.

The lack of evidence for significant ameliorative or transformative social justice impacts in these photovoice studies diminishes the social justice promise of this methodological tool. Photovoice use may indirectly further marginalize participants through attributional bias. Attributional bias may occur, for example, when images and text are used to locate social justice problems within disadvantaged communities, but not in advantaged communities (Lin and Harris 2008). There is a need for a deeper discussion of research ethics concerning such bias in future reviews of photovoice studies. Publication bias is also an issue to consider as high impact journals may be less likely to publish photos.

Despite the notion of the underlying CBPR approach in many of the studies reviewed, researchers did not make transparent the community-based aspect of their study. As emphasized by O'Toole et al. (2003), it is important to distinguish between community-based and community-placed research, as the latter often constitute an imbalance between the research process and targeted outcomes. Pioneers of CBPR have cautioned researchers to clearly provide evidence of the appropriate use of CBPR in their methodologies (Burhansstipanov, Christopher and Schumacher 2005; Strickland 2006).

In an age where digital images are omnipresent, the use of participant photography in qualitative research has become accessible and commonplace. This review has shown that as the use of this method expands, important methodological concerns persist for researchers whose critical theoretical research designs are committed to the significant advancement of social justice. In particular, researchers must 
consider not only the extent of participation in all aspects of the study (as suggested by Catalani and Minkler) but also the extent to which social justice awareness, amelioration or transformation may be achieved.

This review suggests the need to continue the conversation regarding photovoice impact from the individual to the systemic level. Another consideration that warrants attention, and was not explored in this paper, is the power relations between researcher and participants. When evaluating the photovoice method, researchers must also ask themselves: Are both participants and researchers becoming aware of the oppressive situations and outcomes in the research process? Are both participants and researchers taking actions to ameliorate and/or transforming the status quo? Is the research method in itself empowering and not caught between the politics involved in the rigidity of research designs and reports? (Evans-Agnew et al. in press). Based on Wang's and Friere's ontological and epistemological stance on conscientization and empowerment, addressing the researcher/advocate dichotomy must not be the only ultimate outcome. Addressing these questions re-engineers action from an individual to a multilevel ecological scale.

\section{Limitations}

This review only critiqued photovoice research studies published between 2008 and 2013. This excluded articles prior to 2008. However, our goal was to build upon Catalani and Minkler (2010)'s review of photovoice with a new direction to describe the social justice intent of photovoice methods in research.

This review depended on data and contexts reported in the research reports. Word limits of journals may have constrained the reporting details. Thus, particular aspects of social justice action may have been omitted in the interests of space. We also did not make an attempt to contact the researchers themselves to discover more contexts and outcomes. Moreover, given the timeframe required for research reports (from initiation to publication), it may be challenging to have already recorded ameliorative and transformative impacts. One way to address this limitation is for researchers to generate follow-up reports on the long-term outcomes and impacts of their photovoice studies.

Participatory studies are often by necessity small, thus transformative impact at the policy/system level may be harder to achieve during the course of the research study. Similarly, small changes made in the contexts of ameliorative impact may not be recorded or observed. Thus, changes may not have been noted in the research articles.

\section{Implication and Recommendations for Nurse Researchers}

Given the nature of the nursing discipline, which strongly emphasize on the wellbeing of vulnerable populations, the photovoice method, if used effectively, can be a great method to advance the science and achieve optimal wellbeing. This review indicated the need for more nurse researchers to target systemic-level impacts of the photovoice method. Certainly, impact at the individual level of awareness-raising is important. However, ameliorative and transformative impacts have significant implications in addressing the contextual social structural factors influencing health and wellbeing. To facilitate ameliorative and transformative impacts, nurse researchers can (i) include policy stakeholders on the research team; (ii) use the participatory approach where participants not only identify the health concern but are also involved in the implementation of interventions addressing the health concern; (iii) explore various avenues to disseminate the photos and text (i.e. forums and social media); (iv) follow up with and assist stakeholders in various actions required to promote social and structural ranges to improve health outcomes.

\section{CONCLUSION}

The original intent for the use of photovoice was to facilitate change. Given its original intent and theoretical underpinnings, the photovoice method aligns well with the social justice framework, which emphasizes the facilitation of just conditions for individual and community wellbeing. However, as suggested by this review, researchers have yet to embrace the full potential of photovoice, and few studies as yet have resulted in system-level change. If researchers identify a clear underlying methodology-method fit (guided by the goal of promoting social justice) and the social justice impact of their research studies using photovoice, the original promise of this method to address social justice and inequality could be achieved.

\section{REFERENCES}

Allen Q. 2012. Photographs and stories: Ethics, benefits and dilemmas of using participant photography with Black middle-class male youth. Qualitative Research 12: 443-58.

Andonian L and A MacRae. 2011. Well older adults within an urban context: Strategies to create and maintain social participation. The British Journal of Occupational Therapy 74: 2-11. 
Bharmal N, D Kennedy, L Jones, C Lee-Johnson, DA Morris, B Caldwell et al. 2012. Through our eyes: Exploring African-American men's perspective on factors affecting transition to manhood. Journal of General Internal Medicine 27: 153-9.

Boutain DM. 2011. Social justice in nursing: A review of the literature. In Caring for the vulnerable, 3rd edn, eds M de Chesnay and BA Anderson, 43-57. Sudbury, MA: Jones \& Barlett Learning.

Brazg T, B Bekemeier, C Spigner and CE Huebner. 2011. Our community in focus: The use of photovoice for youth-friven dubstance sbuse sssessment and health promotion. Health Promotion Practice 12: 502-11.

Buettner-Schmidt K and ML Lobo. 2012. Social justice: A concept analysis. Journal of Advanced Nursing 68: 948-58.

Burhansstipanov L, S Christopher and SA Schumacher. 2005. Lessons learned from community-based participatory research in Indian country. Cancer Control 12 (Suppl 2): $70-6$.

Carlson ED, J Engebretson and RM Chamberlain. 2006. Photovoice as a social process of critical consciousness. Qualitative Health Research 16: 836-52.

Castleden H, T Garvin and H First Nation. 2008. Modifying hotovoice for community-based participatory indigenous research. Social Science E Medicine 66: 1393-405.

Catalani C and M Minkler. 2010. Photovoice: A review of the literature in health and public health. Health Education $\mathcal{E}$ Behavior 37: 424-52.

Chenail RJ. 2009. Communicating your qualitative research better. Family Business Review 22: 105-8.

Chilton M, J Rabinowich, C Council and J Breaux. 2009. Witnesses to hunger: Participation through photovoice to ensure the right to food. Health and Human Rights 11: 73-85.

Corbin J and A Strauss. 1990. Basics of qualitative research: Grounded Theory procedures and techniques. Thousand Oaks, CA: Sage.

Crotty M. 1998. The foundations of social research: Meaning and perspective in the research process. London, Thousand Oaks, CA: Sage.

D’Alonzo KT. 2010. Getting started in CBPR: Lessons in building community partnerships for new researchers. Nursing Inquiry 17: 282-8.

Davison CM, LM Ghali and P Hawe. 2011. Insights into the school environment that surveys alone might miss: An exploratory pilot study using photovoice. Advances in School Mental Health Promotion 4: 44-51.

Denov M, D Doucet and A Kamara. 2012. Engaging war affected youth through photography: Photovoice with former child soldiers in Sierra Leone. Intervention 10: 117-33.

Downey LH, CL Ireson and FD Scutchfield. 2009. The use of photovoice as a method of facilitating deliberation. Health Promotion Practice 10: 419-27.

Drevdahl D, SM Kneipp, MK Canales and KS Dorcy. 2001. Reinvesting in social justice: A capital idea for public health nursing? Advances in Nursing Science 24: 19-31.

Duffy L. 2010. Hidden heroines: Lone mothers assessing community health using photovoice. Health Promotion Practice 11: 788-97.

Evans-Agnew R, MA Sanon and D Boutain. In press. Critical research methodologies and social justice issues: A methodological example using photovoice. In Philosophies and practices of emancipatory nursing: Social justice as praxis, eds PN Kagan, MC Smith and PL Chinn, Routledge.

Findholt NE, YL Michael and MM Davis. 2011. Photovoice engages rural youth in childhood obesity prevention. Public Health Nursing 28: 186-92.

Flum MR, CE Siqueira, A DeCaro and S Redway. 2010. Photovoice in the workplace: A participatory method to give voice to workers to identify health and safety hazards and promote workplace change - a study of university custodians. American Journal of Industrial Medicine 53: 1150-8.

Foster-Fishman P, B Nowell, Z Deacon, MA Nievar and P McCann. 2005. Using methods that matter: The impact of reflection, dialogue, and voice. American Journal of Community Psychology 36: 275-91.

Foster-Fishman PG, KM Law, LF Lichty and C Aoun. 2010. Youth ReACT for social change: A method for youth participatory action research. American Journal of Community Psychology 46: 67-83.

Freire P. 1973. Education for critical consciousness. New York, NY: Seabury.

Ganong LH. 1987. Integrative reviews of nursing research. Research in Nursing $\mathcal{E}^{\circ}$ Health 10: 1-11.

Green E and B Kloos. 2009. Facilitating youth participation in a context of forced migration: A photovoice project in northern Uganda. Journal of Refugee Studies 22: 460-82.

Grieb SMD, RM Joseph, A Pridget, H Smith, R Harris and J Ellen. 2013. Understanding housing and health through the lens of transitional housing members in a high-incarceration Baltimore City neighborhood: The GROUP Ministries Photovoice Project to promote community redevelopment. Health E Place 21: 20-8.

Halifax NVD, F Yurichuk, J Meeks and E KhandorE. 2008. Photovoice in a Toronto community partnership: Exploring the social determinants of health with home- 
less people. Progress in Community Health Partnerships 2: 129-36.

Hannay J, R Dudley, S Milan and PK Leibovitz. 2013. Combining photovoice and focus groups: Engaging Latina teens in community assessment. American Journal of Preventive Medicine 44 (Suppl. 3): S215-24.

Hansen-Ketchum P and F Myrick. 2008. Photo methods for qualitative research in nursing: An ontological and epistemological perspective. Nursing Philosophy 9: 20513.

Harper K. 2012. Visual interventions and the "crises in representation" in environmental anthropology: Researching environmental justice in a Hungarian Romani neighborhood. Human Organization 71: 292-305.

Haque N and B Eng. 2011. Tackling inequity through a photovoice project on the social determinants of health: Translating photovoice evidence to community action. Global Health Promotion 18: 16-19.

Hergenrather KC, SD Rhodes, CA Cowan, G Bardhoshi and S Pula. 2009. Photovoice as community-based participatory research: A qualitative review. American Journal of Health Behavior 33: 686-98.

Kirkham SR and JM Anderson. 2002. Postcolonial nursing scholarship: From epistemology to method. Advances in Nursing Science 25: 1-17.

Kramer L, P Schwartz, A Cheadle, JE Borton, M Wright, C Chase et al. 2010. Promoting policy and environmental change using photovoice in the Kaiser Permanente Community Health Initiative. Health Promotion Practice 11: 332-9.

Lardeau M, G Healey and J Ford. 2011. The use of photovoice to document and characterize the food security of users of community food programs in Iqaluit, Nunavut. Rural and Remote Health 11: 1-17.

Lin AC and DR Harris. 2008. The colors of poverty: Why racial and ethnic disparities persist. New York, NY: Russell Sage Foundation.

Lorenz LS and B Kolb. 2009. Involving the public through participatory visual research methods. Health Expectations 12: $262-74$.

Mack L. 2010. The philosophical underpinnings of educational research. Polyglossia 19: 5-11.

Markus SF. 2012. Photovoice for healthy relationships: Community-based participatory HIV prevention in a rural American Indian community. American Indian and Alaska Native Mental Health Research 19: 102-23.

Mohammed S, SZ Sajun and FS Khan. 2013. Harnessing photovoice for tuberculosis advocacy in Karachi, Pakistan. Health Promotion International. DOI: 10.1093/ heapro/dat036 [Epub ahead of print].
Newman SD. 2010. Evidence-based advocacy: Using photovoice to identify barriers and facilitators to community participation after spinal cord injury. Rehabilitation Nursing 35: 47-59.

Novek S and T Morris-Oswald. 2012. Using photovoice with older adults: Some methodological strengths and issues. Ageing and Society 32: 451-70.

O'Toole TP, KF Aaron, MH Chin, C Horowitz and F Tyson. 2003. Community-based participatory research. Journal of General Internal Medicine 18: 592-4.

Plunkett R, BD Leipert and SL Ray. 2013. Unspoken phenomena: Using the photovoice method to enrich phenomenological inquiry. Nursing Inquiry 20: 156-64.

Ponic P, C Reid and W Frisby. 2010. Cultivating the power of partnerships in feminist participatory action research in women's health. Nursing Inquiry 17: 324-35.

Poudrier J and RT Mac-Lean. 2009. 'We've fallen into the cracks': Aboriginal women's experiences with breast cancer through photovoice. Nursing Inquiry 16: 306-17.

Prins E. 2010. Participatory photography: A tool for empowerment or surveillance? Action Research 8: 426-43.

Pritzker S, A LaChapelle and J Tatum. 2012. "We need their help": Encouraging and discouraging adolescent civic engagement through photovoice. Children $\mathcal{F}^{\circ}$ Youth Services Review 34: 2247-54.

Redman RW and L Clark. 2002. Service-learning as a model for integrating social justice in the nursing curriculum. Journal of Nursing Education 41: 446-9.

Royce SW, D Parra-Medina and DH Messias. 2006. Using photovoice to examine and initiate youth empowerment in community-based programs: A picture of process and lessons learned. Californian Journal of Health Promotion 4: 80-91.

Seitz CM, RW Strack, R Rice, E Moore, T DuVall and DL Wyrick. 2012. Using the photovoice method to advocate for change to a campus smoking policy. Journal of American College Health 60: 537-40.

Strickland CJ. 2006. Challenges in community-based participatory research implementation: Experiences in cancer prevention with Pacific Northwest American Indian tribes. Cancer Control 13: 230-6.

Tanjasiri SP, R Lew, DG Kuratani, M Wong and L Fu. 2011. Using photovoice to assess and promote environmental approaches to tobacco control in AAPI communitiesr. Health Promotion Practice 12: 654-65.

Valera P, J Gallin, D Schuk and N Davis. 2009. "Trying to eat healthy": A photovoice study about women's access to healthy food in New York City. Affilia Journal of Women $\mathcal{E}$ Social Work 24: 300-14. 
Vaughn LM, JR Forbes and B Howell. 2009. Enhancing home visitation programs: Input from a participatory evaluation using photovoice. Infants E Young Children 22: 132-45.

Wang C and MA Burris. 1994. Empowerment through photo novella: Portraits of participation. Health Education $\mathcal{G}^{\circ}$ Behavior 21: 171-86.

Wang CC and MA Burris. 1997. Photovoice: Concept, methodology, and use for participatory needs assessment. Health Education E Behavior 24: 369-87.

Wang CC. 1999. Photovoice: A participatory action research strategy applied to women's health. Journal of Women's Health 8: 185-92.
Wang CC and CA Pies. 2004. Family, maternal, and child health through photovoice. Maternal $\mathcal{E}$ Child Health Journal 8: 95-102.

Wang C and YA Redwood-Jones. 2001. Photovoice ethics: Perspectives from Flint photovoice. Health Education $\mathcal{E}$ Behavior 28: 560-72.

Wang CC, S Morrel-Samuels, PM Hutchison, L Bell and RM Pestronk. 2004. Flint photovoice: Community building among youths, adults, and policymakers. American Journal of Public Health 94: 911-3.

Weiler K. 1988. Women teaching for change: Gender, class and power. New York, NY: Bergin \& Garvey. 